\title{
Do different muscle strength levels affect stability during unplanned gait termination?
}

\author{
XUANZHEN CEN ${ }^{1}$, XINYAN JIANG $^{1}$, YAODONG GU $^{1,2 *}$ \\ ${ }^{1}$ Faculty of Sports Science, Ningbo University, Ningbo, China. \\ ${ }^{2}$ Auckland Bioengineering Institute, University of Auckland, Auckland, New Zealand.
}

\begin{abstract}
Purpose: Unplanned gait termination (UGT) widely occurs in various sports and daily life as a kind of stress response to unexpected stimulus. However, the body stability may be greatly affected when the body completely stops. The purpose of this study was to examine the association between muscle strength levels and body stability during UGT through comparing the plantar pressure. Methods: Twenty healthy participants (10 male and 10 female) with different lower limbs muscle strength and power were asked to perform planned gait termination (PGT) and unplanned gait termination (UGT) on an 8-m walkway. Related plantar pressure data including maximum pressure, maximum force, contact area and center of pressure were recorded with Footscan pressure platform. Results: Two types of gait termination have significant differences in the plantar pressure distribution. Maximum pressure and maximum force in the lateral metatarsal increased significantly during UGT, compared to PGT. At the same time, data from the current study suggested that there might be a correlation between the muscle strength levels of individual and the stability during the gait termination, especially between the muscle power and UGT, which means that the more excellent muscle power an individual has, the more stable the body is when UGT is performed. Conclusions: The findings suggest that different muscle strength levels could affect stability during unplanned gait termination.
\end{abstract}

Key words: muscle power, muscle strength, gait stop, plantar pressure, foot balance

\section{Introduction}

Human walking is a continuous process from initiation to termination, which basic requirement is to keep the body stabile, and to complete the position movement [21]. A large number of studies have been done on the first two parts, e.g., influence of external disturbance [8], [12], related diseases [7], [25] and demographic factors [11], [17]. The gait termination (GT), which is defined as the transition from a dynamic state to static state of equilibrium at the level of space-time [21], is also an important research topic. The mechanism of GT is divided into increasing braking force, decreasing push-off force and converting kinetic energy into potential energy [9].
According to different control processes, GT can be divided into planned gait termination (PGT) and unplanned gait termination (UGT). PGT means to efficiently reach the expected position or nearby in term of the purpose of movement under the dual restriction of human and environment [2], [20]. The baserunning of baseball players is a good example. UGT is a stress response to unexpected stimulus, and the body stability is greatly affected when the complete stop [20]. For instance, in table tennis, athletes often stop the original moving route and move in the opposite direction to cater to the returning table tennis because the direction of the opponent's strike is unknown. Whether the received termination instruction can be executed quickly and effectively is crucial to individual's safety [18].

\footnotetext{
* Corresponding author: Yaodong Gu, Faculty of Sports Science, Ningbo University. No. 818, Fenghua Road, Jiangbei District, Ningbo, Zhejiang, China. 315211. E-mail: guyaodong@hotmail.com

Received: July 17th, 2019

Accepted for publication: September 17th, 2019
} 
Based on the above-mentioned terminating mechanisms, when it is necessary to stop urgently, a net braking impulse is needed to adapt new situation of body posture [3]. Previous studies [2], [19] have shown that UGT with preferred speed and PGT with fast speed have similar mechanic which in peak knee and hip flexion angles were not statistically significantly different. UGT does not have a buffering process in the face of sudden stimuli, as opposed to PGT that has a full deceleration process before reaching the target position [24]. This means that the walking speed before UGT is faster than PGT, and walkers often are more difficult to complete the gait stop as walking speed increases [2]. When the human is in normal movement, the body will be in a state of dynamic balance, but when the unexpected stimulation forces it to stop the gait suddenly, the previous dynamic balance will be destroyed, and the stability of the walker's body posture will be seriously affected. At the same time, the body will take a series of adaptation to minimize this imbalance to avoid falls, e.g., faster rate of muscle activation [1] and muscle recruitment [23].

Sarah et al. [18] demonstrated that most people stabilize and complete the stopping task by a strategy of hip/knee extension or hip/knee flexion during UGT, in addition, the ability to stop quickly and safely depends largely on the ability to create sufficient joint moments in a short period of time. In their other study [19], subjects revealed extra range of motion and muscle strength required in the knee joints during UGT. Moreover, stretching and strengthening the related muscles of the lower extremity, such as the quadriceps and hamstrings, could contribute to maintain position balance during termination. Other studies have shown that people with conditions such as Parkinson's disease, which can lead to a loss of muscle strength, can have a serious impact on posture stability during UGT [1], [16]. To the best of our knowledge, at present, studies about UGT mainly focus on kinematics, while kinetics studies are limited to the joint moment of lower limbs. Plantar pressure data have been validated by previous studies as an indicator of human stability in different populations and under different conditions [22], [27], [29]. However, there is no relevant plantar pressure study about UGT, and no direct study has compared differences of plantar loading distribution during two types of gait stop and conducted to ascertain whether better lower limbs muscle strength level can lead better stability.

The main purpose of the present study was to examine the correlation between muscle strength levels and body stability during UGT through comparing the plantar pressure. We hypothesized that individual with better muscle strength and power will lead to better stability during UGT.

\section{Materials and methods}

\subsection{Participants}

Twenty healthy students of Ningbo University were selected as experimental subjects including 10 male (age: $24.2 \pm 0.63$ years; weight: $67.7 \pm 4.66 \mathrm{~kg}$; height: $175.6 \pm 3.10 \mathrm{~cm}$; BMI: $21.95 \pm 1.36$ ) and 10 female (age: $23.8 \pm 0.79$ years; weight: $54.8 \pm 2.30 \mathrm{~kg}$; height: $161.1 \pm 3.63 \mathrm{~cm}$; BMI: $20.12 \pm 0.81)$. Since the termination signal of this experiment is received by the participants' auditory organ through the ringing of the bell, the person with hearing impairment was excluded in the selection of the subject. There was no damage to their lower limbs in the first half year of the experiment. The subjects understood the procedure and purpose of the experiment and signed the agreement.

\subsection{Lower limbs muscle strength}

Lower limbs muscle power is the product of speed and force of muscle contraction. And it usually is measured in the leg extensor muscles [16]. Leg extensor muscle strength and power of participants were measured using pneumatic variable resistance training equipment (Keiser Air250, Model Keizer Corp, Fresno, CA, USA) (Fig. 1a). This maximal resistance was defined as one repetition maximum (1RM) represented muscle strength. The test flow of muscle strength is shown in Fig. 1b, following the guideline provided by the National Strength and Conditioning Association (NSCA) [5]. As far as possible, the 1RM was measured less than 5 tests for all subjects, so as to avoid fatigue affecting the accuracy of the test. After the measurement of muscle strength, according to the 1RM of each subject, the maximum power of 7 relative resistances $(30$, $40,50,60,70,80$, and $90 \% 1 \mathrm{RM})$ were tested, respectively [14]. Different resistances appeared randomly, and there was a one-minute rest time between the two tests. Finally, the maximum muscle strength and power were output by comparison, and the two groups of data of the subjects were divided into three grades: Stronger, General and Weaker (Table 1). The male/ female stronger and weaker groups were defined based on $\geq 0.5 \mathrm{SD}$ and $\leq 0.5 \mathrm{SD}$ from the mean muscle strength/ power. 


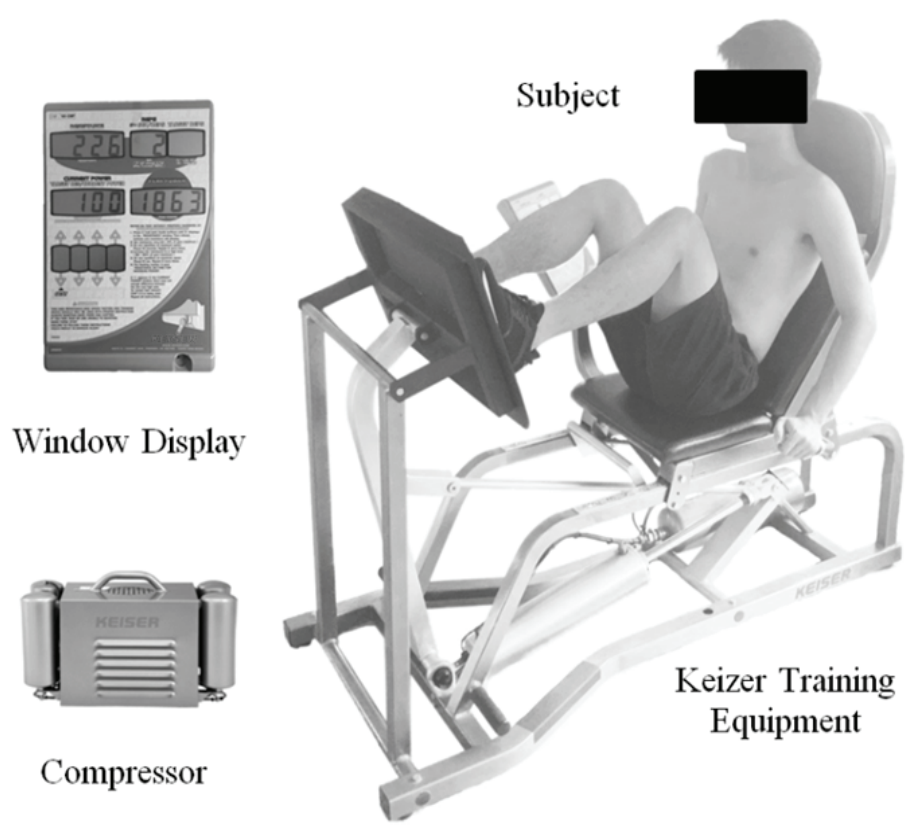

(a)

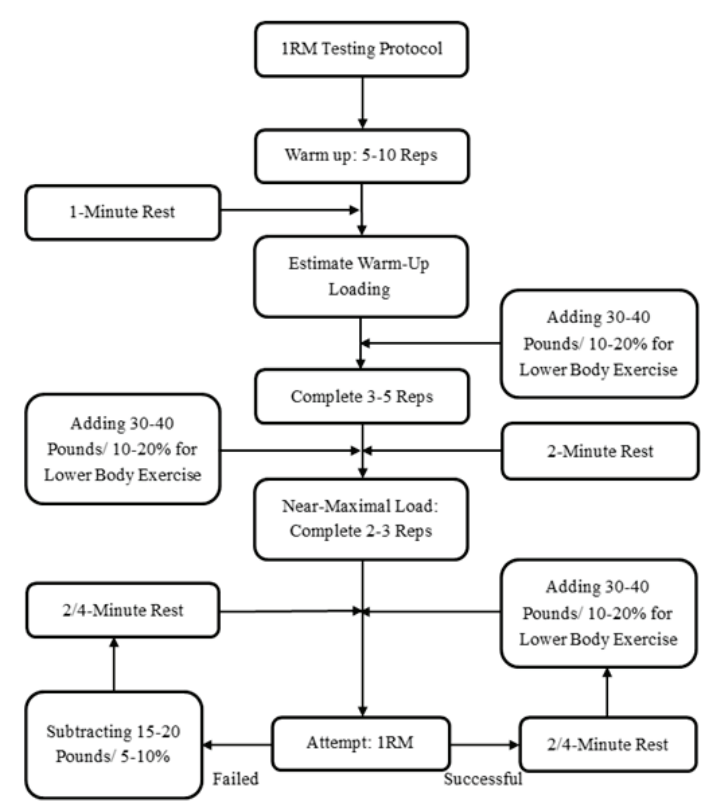

(b)

Fig. 1. The use of Keizer training equipment (a) and the flow chart of 1RM testing protocol (b)

Table 1. Grouping of muscle strength levels of subjects $(\mathrm{M} \pm \mathrm{SD})$

\begin{tabular}{|l|l|c|c|}
\hline & & Muscle Strength [lb] & Muscle Power [W] \\
\hline \multirow{4}{*}{ Male } & Stronger & $695.25 \pm 18.25$ & $2365.00 \pm 225.89$ \\
\cline { 2 - 4 } & General & $581.00 \pm 26.87$ & $1884.67 \pm 124.68$ \\
\cline { 2 - 4 } & Weaker & $481.25 \pm 28.79$ & $1516.50 \pm 112.95$ \\
\hline \multirow{4}{*}{ Female } & Stronger & $551.67 \pm 49.07$ & $1461.67 \pm 193.67$ \\
\cline { 2 - 4 } & General & $433.00 \pm 15.47$ & $1206.75 \pm 71.38$ \\
\cline { 2 - 4 } & Weaker & $381.67 \pm 22.55$ & $1040.00 \pm 87.61$ \\
\hline
\end{tabular}

\subsection{Protocol}

Subjects were asked to walk along an $8 \mathrm{~m}$ walkway at their self-selected speed. The walkway has an integrated $2 \mathrm{~m}$ Footscan pressure plate (RSscan International, Belgium) which was artificially divided into four areas $\mathrm{A}$, $\mathrm{B}, \mathrm{C}$ and $\mathrm{D}$. The size of each area was set according to the area of the Footscan pressure plate (each area was about $50 \mathrm{~cm} \times 50 \mathrm{~cm}$ ). They used the dominant leg (right leg) and the non-dominant leg (left leg) to pass through area A and B respectively, and finally stopped at area D. In the type two, the subjects should stop as quickly and safely as possible in area B if they receive the termination signal as right heel touching area A (Fig. 2). The instructions were sent to the subjects by the staff ringing the bell randomly. In order to reduce the experimental error, the bell ringing work of all tests in this study was completed by the same staff. At the same time, there was a two-minute rest time between the two trials to avoid fatigue affecting the accuracy of the experiment. The

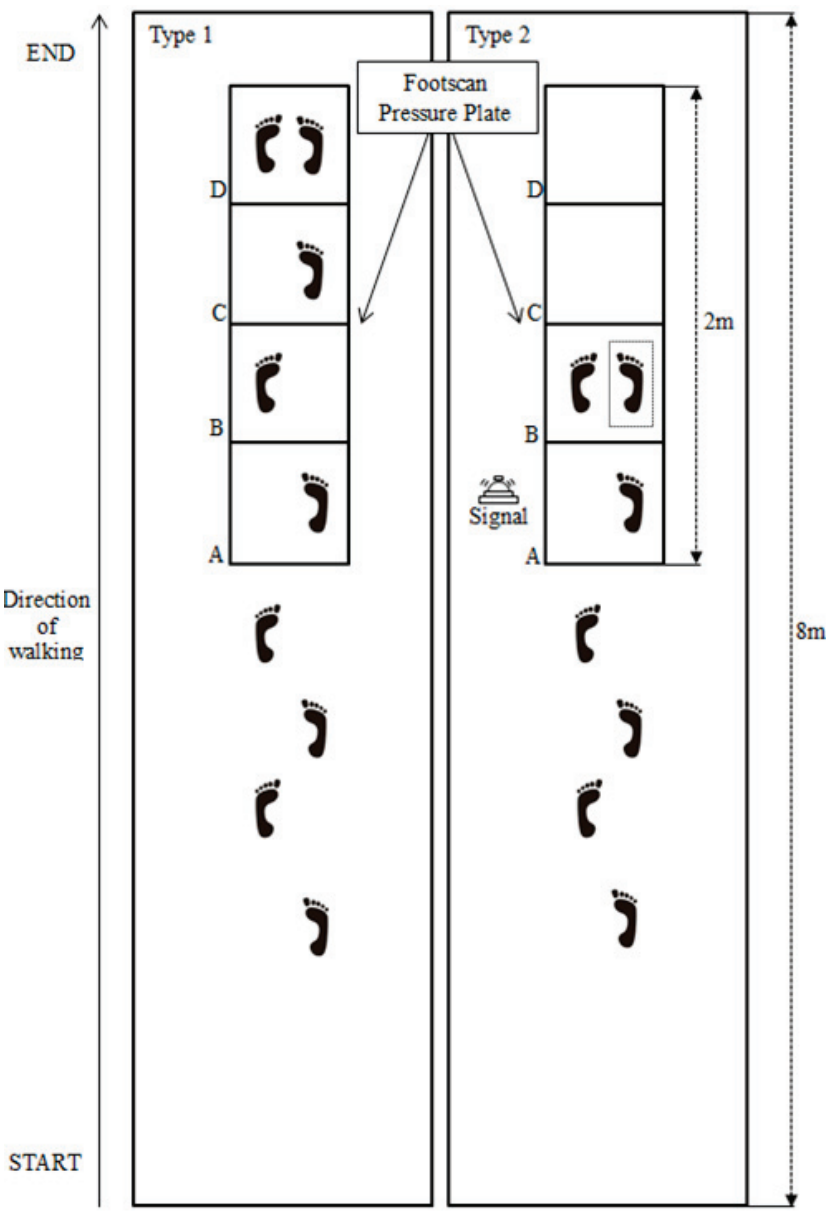

Fig. 2. Overhead view of Footscan pressure plate used for two types of gait termination trials 
pressure plate was used to collect plantar pressure data including COP trajectory of the left foot at the gait stop of the two types. Ten successful experimental data including 5 type 1 and 5 type 2 were required to collect about each subject.

\subsection{Data analysis}

For each gait experiments, ten anatomical regions were artificially divided by the Footscan ${ }^{\circledR} 7.0$ (RsScan International, Belgium), including Big Toe (BT), Other Toes (OT), First Metatarsal (M1), Second Metatarsals (M2), Third Metatarsal (M3), Fourth Metatarsal (M4), Fifth Metatarsal (M5), Midfoot, Medial Heel (MH) and Lateral Heel (LH). Dynamic plantar pressure data were measured comprehensively by high frequency digital sampling technique. Maximal pressure, maximal force and contact area were measured to evaluate the variability of plantar pressure distribution during two types of gait termination experiments. Previous studies [27], [28] have shown that the displacement of the medial-lateral center of pressure (ML-COP) could be used to assess the medial-lateral stability, especially during the forefoot contact phase and the foot flat phase. Therefore, we calculated the range of the ML-COP trajectory by comparing the absolute difference between the largest and smallest coordinate of the COP respectively during these phases. Meanwhile, in this study, foot balance data were collected by calculating force difference between the corresponding anatomical regions. The foot balance was calculated by comparing the medial part of the foot (M1 $+\mathrm{M} 2+\mathrm{MH})$ with the lateral part of the foot $(\mathrm{M} 3+\mathrm{M} 4$ $+\mathrm{M} 5+\mathrm{LH})$. A positive value indicates foot pronation while a negative value indicates foot supination [26].

Independent-samples $t$-tests were used to analyze differences between two kinds of gait termination. When data were non-normally distributed, Mann-Whitney $U$-test was applied. And a Spearman test was used to identify correlations between muscle strength level and body stability during gait termination. All statistical analyses were performed with SPSS 19.0 for Windows ${ }^{\mathrm{TM}}$ software (IBM, Armonk, NY, USA). The significance level was set to $p<0.05$.

\section{Results}

\subsection{Plantar pressure distribution}

The detail plantar pressure data included maximum pressure, maximum force and contact area in BT, OT, M1, M2, M3, M4, M5, MF, MH and LH, which is shown in Tables 2-3 and in Fig. 3.

For male subjects, compared to PGT, all of plantar pressure data showed an increased trend when executing UGT. In aspects of maximum pressure and maximum force, data were increased significantly in OT $(p=0.005, p=0.015)$, M2 $(p=0.004, p=$ $0.006), \mathrm{M} 3(p=0.001, p=0.008), \mathrm{M} 4(p<0.001, p$ $=0.012), \mathrm{M} 5(p<0.001, p=0.003), \mathrm{MF}(p=0.044$, $p=0.041), \mathrm{MH}(p<0.001, p<0.001)$ and LH $(p<$ $0.001, p<0.001)$. However, it was observed that no significant change occurred in any parameter for the BT and M1. In addition, no significant difference were observed in the most of the regions with only the in BT during UGT exhibiting significance increase $(p=0.028)$ in contact area. Similarly, significant increases were exhibited in maximum pressure and maximum force in female subjects during UGT, which are in BT $(p=0.017, p=0.024)$, OT $(p=0.001, p=0.012)$, M2 $(p=0.015, p=0.016), \mathrm{M} 3(p=0.002, p<0.001)$,

Table 2. The male plantar pressure during PGT and UGT

\begin{tabular}{|l|c|c|c|c|c|c|c|c|c|}
\hline \multirow{2}{*}{ Regions } & \multicolumn{3}{|c|}{ Max Pressure Mean (SD) } & \multicolumn{3}{c|}{ Max Force Mean (SD) } & \multicolumn{3}{c|}{ Contact Area Mean (SD) } \\
\cline { 2 - 10 } & PGT & UGT & $P$ value & PGT & UGT & $P$ value & PGT & UGT & $P$ value \\
\hline BT & $2.95(2.55)$ & $3.01(1.68)$ & 0.552 & $42.78(39.65)$ & $47.05(26.86)$ & 0.227 & $13.76(2.72)$ & $15.41(3.59)$ & $0.028^{*}$ \\
\hline OT & $0.40(0.36)$ & $0.66(0.48)$ & $0.005^{*}$ & $5.32(5.29)$ & $9.09(8.45)$ & $0.015^{*}$ & $11.29(4.82)$ & $13.43(4.74)$ & 0.053 \\
\hline M1 & $3.04(1.89)$ & $3.71(1.78)$ & 0.119 & $39.07(26.65)$ & $52.84(31.39)$ & 0.051 & $13.01(3.05)$ & $12.99(2.81)$ & 0.981 \\
\hline M2 & $5.29(2.11)$ & $6.71(2.25)$ & $0.004^{*}$ & $67.74(28.27)$ & $85.45(29.06)$ & $0.006^{*}$ & $12.23(1.89)$ & $12.43(1.20)$ & 0.578 \\
\hline M3 & $6.21(2.17)$ & $8.50(3.30)$ & $0.001^{*}$ & $69.28(27.67)$ & $91.90(40.90)$ & $0.008^{*}$ & $10.10(1.34)$ & $10.38(1.12)$ & 0.336 \\
\hline M4 & $4.96(1.48)$ & $7.10(2.64)$ & $0.000^{*}$ & $57.38(20.40)$ & $73.34(29.38)$ & $0.012^{*}$ & $10.08(1.72)$ & $10.43(0.81)$ & 0.245 \\
\hline M5 & $2.82(1.11)$ & $4.40(1.82)$ & $0.000^{*}$ & $37.15(18.87)$ & $52.99(25.05)$ & $0.003^{*}$ & $11.41(2.39)$ & $12.37(1.97)$ & 0.067 \\
\hline MF & $1.76(0.65)$ & $2.08(0.76)$ & $0.044^{*}$ & $52.50(28.15)$ & $64.63(28.90)$ & $0.041^{*}$ & $27.04(6.77)$ & $29.03(6.49)$ & 0.298 \\
\hline MH & $7.89(3.09)$ & $12.90(5.50)$ & $0.000^{*}$ & $189.98(83.26)$ & $283.37(105.41)$ & $0.000^{*}$ & $21.80(3.49)$ & $21.93(2.49)$ & 0.841 \\
\hline LH & $7.16(2.57)$ & $11.37(4.46)$ & $0.000^{*}$ & $158.29(55.17)$ & $240.39(94.05)$ & $0.000^{*}$ & $20.24(2.86)$ & $21.10(3.05)$ & 0.207 \\
\hline
\end{tabular}

Notes: * Significant difference, $p<0.05$. 
Table 3. The female plantar pressure during PGT and UGT

\begin{tabular}{|l|c|c|c|c|c|c|c|c|c|}
\hline \multirow{2}{*}{ Regions } & \multicolumn{2}{|c|}{ Max Pressure Mean (SD) } & \multicolumn{3}{c|}{ Max Force Mean (SD) } & \multicolumn{3}{c|}{ Contact Area Mean (SD) } \\
\cline { 2 - 10 } & PGT & UGT & $P$ value & PGT & UGT & $P$ value & PGT & UGT & $P$ value \\
\hline BT & $2.02(1.68)$ & $3.02(1.89)$ & $0.017^{*}$ & $22.45(20.53)$ & $35.48(26.14)$ & $0.024^{*}$ & $10.19(2.66)$ & $11.32(3.12)$ & 0.096 \\
\hline OT & $0.41(0.39)$ & $0.81(0.60)$ & $0.001^{*}$ & $4.67(4.55)$ & $9.16(9.04)$ & $0.012^{*}$ & $9.67(6.09)$ & $12.15(6.20)$ & 0.083 \\
\hline M1 & $2.64(1.76)$ & $3.32(2.35)$ & 0.282 & $23.77(17.34)$ & $32.04(24.80)$ & 0.210 & $9.37(2.25)$ & $10.32(2.58)$ & 0.052 \\
\hline M2 & $5.19(1.68)$ & $6.33(2.30)$ & $0.015^{*}$ & $52.12(19.81)$ & $64.19(22.55)$ & $0.016^{*}$ & $9.65(1.02)$ & $10.49(1.29)$ & $0.002^{*}$ \\
\hline M3 & $6.74(1.34)$ & $8.33(2.73)$ & $0.002^{*}$ & $56.15(13.13)$ & $72.13(24.11)$ & $0.000^{*}$ & $8.22(0.86)$ & $8.84(1.11)$ & $0.012^{*}$ \\
\hline M4 & $5.29(1.34)$ & $7.02(1.97)$ & $0.000^{*}$ & $41.88(11.68)$ & $60.00(17.06)$ & $0.000^{*}$ & $8.29(1.03)$ & $8.78(0.99)$ & $0.037^{*}$ \\
\hline M5 & $3.03(0.93)$ & $3.84(1.38)$ & $0.005^{*}$ & $29.02(11.13)$ & $40.40(17.19)$ & $0.002^{*}$ & $9.24(1.43)$ & $9.88(1.62)$ & 0.070 \\
\hline MF & $2.53(0.96)$ & $3.09(1.60)$ & 0.069 & $71.60(37.87)$ & $89.42(54.53)$ & 0.101 & $27.85(5.48)$ & $29.11(4.65)$ & 0.283 \\
\hline MH & $9.54(2.80)$ & $13.50(4.06)$ & $0.000^{*}$ & $144.84(44.75)$ & $217.99(72.15)$ & $0.000^{*}$ & $15.76(1.57)$ & $16.25(1.61)$ & 0.209 \\
\hline LH & $8.68(3.02)$ & $11.66(3.49)$ & $0.000^{*}$ & $133.43(50.68)$ & $181.12(53.99)$ & $0.000^{*}$ & $15.44(1.71)$ & $15.71(1.45)$ & 0.474 \\
\hline
\end{tabular}

Notes: * Significant difference, $p<0.05$.

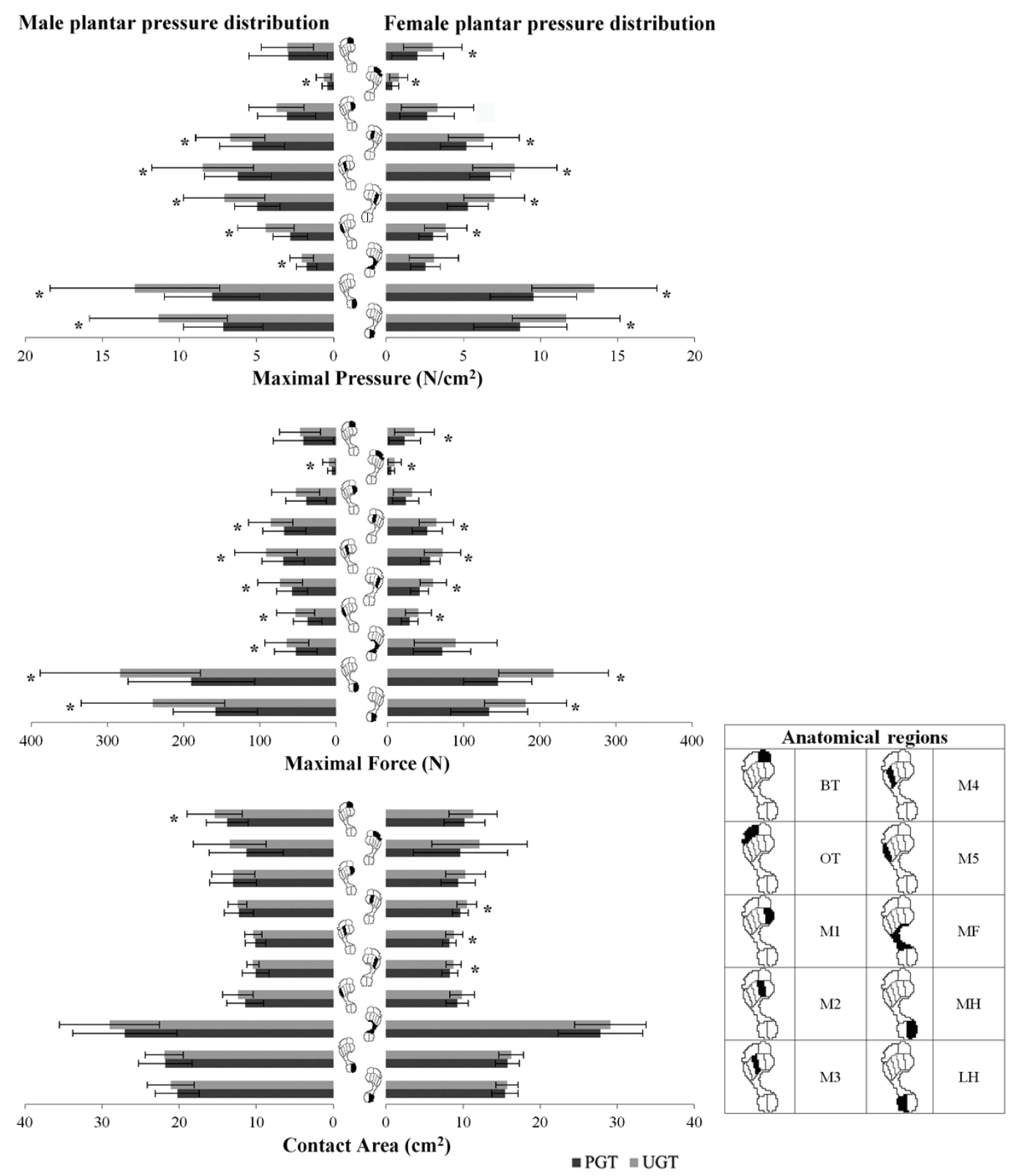

Fig. 3. The plantar pressure during PGT and UGT.

Notes: * Significant difference, $p<0.05$ 
M4 $(p<0.001, p<0.001)$, M5 $(p=0.005, p=0.002)$, $\mathrm{MH}(p<0.001, p<0.001)$ and LH $(p<0.001$, $p<0.001)$. The difference in contact area mainly focuses on M2 ( $p=0.002)$, M3 $(p=0.012)$ and M4 $(p=0.037)$, and both of them show an increasing trend in UGT, compared to PGT.

\subsection{Foot balance}

The foot balance data and trajectories of COP during two types of gait termination are shown in Table 4. From maximum, minimum and range, all subjects had

Table 4 . The stability of two types of gait termination

\begin{tabular}{|l|l|c|c|c|c|c|c|}
\hline \multicolumn{4}{|c|}{} & \multicolumn{2}{c|}{ Male } & \multicolumn{2}{c|}{ Female } \\
\cline { 2 - 8 } & & PGT & UGT & $P$ value & PGT & UGT & $P$ value \\
\hline \multirow{3}{*}{ Foot Balance } & Max & $1.49(1.21)$ & $2.63(1.34)$ & $0.000^{*}$ & $0.95(0.78)$ & $2.12(2.25)$ & 0.077 \\
\cline { 2 - 8 } & Min & $-2.45(1.30)$ & $-3.17(1.75)$ & $0.038^{*}$ & $-2.59(1.23)$ & $-3.93(2.13)$ & $0.001^{*}$ \\
\cline { 2 - 8 } & Range & $3.94(1.52)$ & $5.80(1.87)$ & $0.000^{*}$ & $3.54(1.09)$ & $6.06(2.76)$ & $0.000^{*}$ \\
\hline \multirow{3}{*}{ ML-COP } & Max & $-6.97(2.75)$ & $-4.85(4.32)$ & $0.006^{*}$ & $-6.96(2.23)$ & $-6.09(2.33)$ & $0.047^{*}$ \\
\cline { 2 - 8 } & Min & $-14.57(2.98)$ & $-16.82(5.40)$ & $0.011^{*}$ & $-14.81(2.34)$ & $-16.94(4.48)$ & $0.016^{*}$ \\
\cline { 2 - 8 } & Range & $7.60(2.56)$ & $11.97(5.61)$ & $0.000^{*}$ & $7.85(2.15)$ & $10.85(5.07)$ & $0.001^{*}$ \\
\hline
\end{tabular}

Notes: * Significant difference, $p<0.05$.
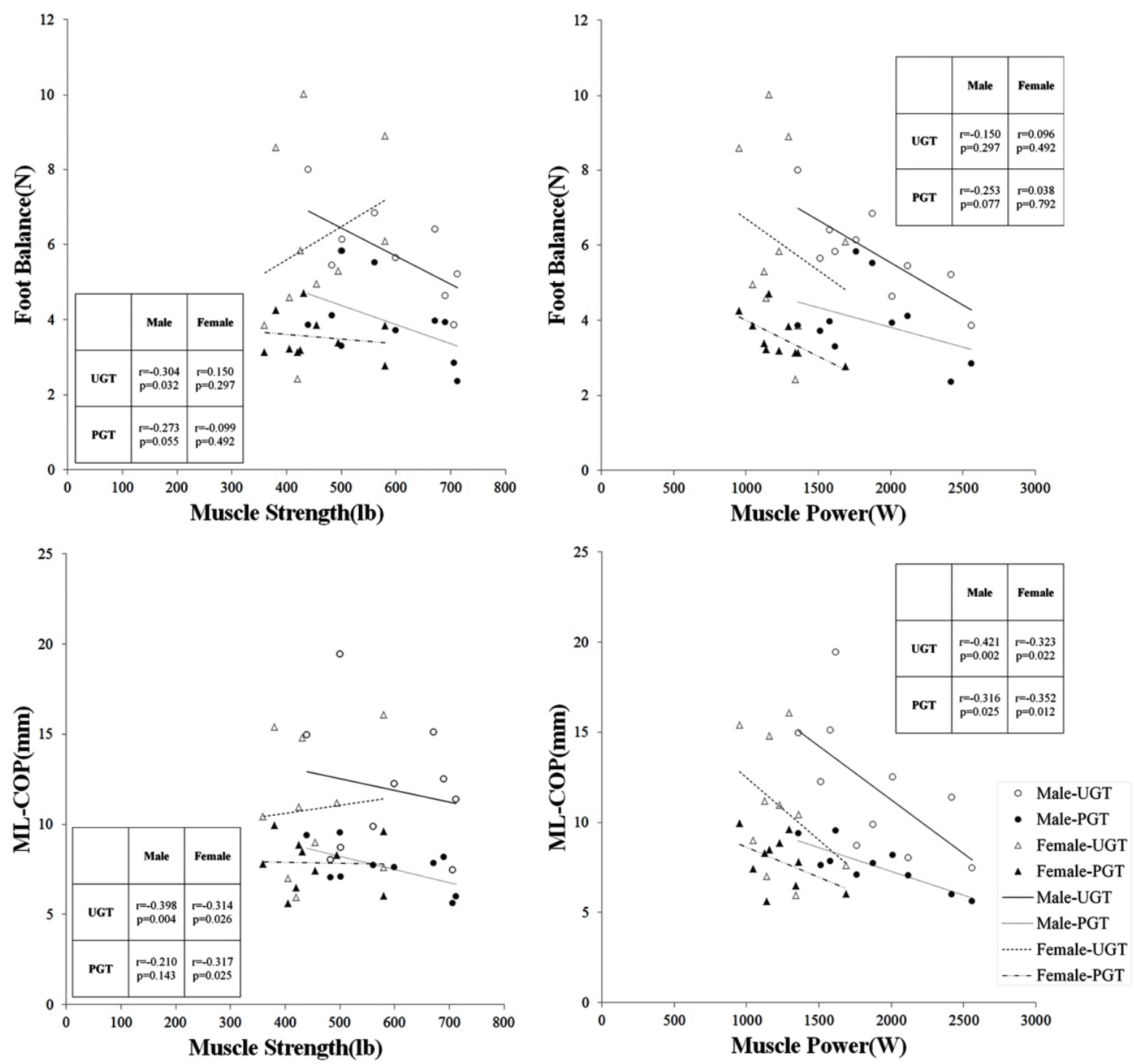

Fig. 4. The Scatterplots of stability and muscle strength levels during two types of gait termination 
significant differences in foot balance and trajectories of ML-COP (all $p$ values $<0.05$ ), except maximum of foot balance in female subjects. And compared to the PGT, the range of foot balance during UGT in male and female subjects were increased by $1.86 \mathrm{~N}(p<0.001)$ and $2.52 \mathrm{~N}(p<0.001)$, respectively. For trajectories of ML-COP, the range during UGT in male and female subjects were increased by $4.37 \mathrm{~mm}(p<0.001)$ and $3.00 \mathrm{~mm}(p=0.001)$, respectively.

In Figure 4, the correlations between muscle strength levels and foot balance are illustrated. The better the muscle strength $(r=-0.304, p=0.032)$ and power $(r=-0.398, p=0.004)$ of the male subjects, the smaller the force difference between medial and lateral part of the foot during UGT. Similarly, along with the increase of muscle strength $(r=-0.150, p=0.297)$ and power $(r=-0.421, p=0.001)$ of male subjects, the range of the ML-COP trajectory was smaller. There are significant correlations between female muscle power and range of foot balance $(r=-0.314, p=0.026)$ and ML-COP $(r=-0.323, p=0.022)$. However, the correlation between foot balance and ML-COP with the muscle strength of female subjects was not significant.

\section{Discussion}

This study compared the plantar pressure variables and identified correlations between muscle strength levels and foot balance during two types of gait stop as well as plantar pressure data including maximum pressure, maximum force, contact area and COP trajectory, and muscle strength level including muscle strength and muscle power.

Our results suggested that maximum pressure and force were increased significantly in middle and lateral metatarsal as well as heel during UGT, compared to PGT. In particular, the imbalance in the increase of metatarsal pressure may lead to a challenge in foot motion during quick stop, which increases risk of overpronation/supination. Although the maximum pressure and force in M1 also show an increasing trend, there is no significant difference, so the cutaneous receptors in the first metatarsal region may not play an effective role in the human body's timely feedback, posture modification and balance maintenance [15]. Although the maximum pressure and force in BT showed an increasing trend in both male and female subjects, the data of female subjects were significant ( $p=0.017, p=0.012)$, which might due to the inability of female subjects to generate sufficient net braking impulse when it is necessary to stop urgently [3],
[9]. Therefore, the center of mass still tends to move forward when they stop, which might lead to high plantar pressure in BT. Chatzistergos et al. [6] found a weak significant correlation between hallux grip force and gait balance, but this was more for people with balance disorders. People usually choose to stop at a stable way during UGT, and they will stop at the metatarsal area in the forefoot contact phase. But UGT is usually fast, and the human body does not have enough reaction time, but for reasons of stability and inertia, the human body will perform a toe landing in forefoot contact phase. The average strength level of women is lower than that of men. Therefore, on the maximum pressure and force, female subjects also show significant differences on BT and OT. It's worth mentioning that the significant increment of contact area in middle and lateral metatarsal of female subjects may be due to the body passively increasing the geometric base of support in response to acute changes in balance [4]. These may also reflect that women are less stable than men during UGT.

Foot balance was reflected by difference value of plantar force between the medial and lateral foot during heel contact, midstance and propulsion phase, and positive and negative values of the difference represent overpronation and underpronation of the foot, respectively [26]. The peak absolute value of overpronation and underpronation of the foot in UGT revealed an increasing trend, compared to in PGT. Especially in the maximum value of foot rotation, this may result in pronation during UGT, which meant that the subjects' foot balance was greater damaged at this time, resulting in an increased risk of joint damage. The foot balance data of female subjects are also worth paying attention, and, compared to male subjects, they are more likely to have the risk of foot supination during UGT. In addition, the variability of full foot balance in two types of gait stop is focused on the range between maximum and minimum force difference, especially in the lateral part of foot. This indicates that the degree of foot over pronation is more obvious during the complete phase during UGT. Changes in ML-COP have been assumed to be one of measurement index of fall risk [13]. The trajectories of COP were mainly located medially to the subtalar joint axis during related phase which would cause the ground reaction force to apply a pronation moment around the subtalar joint [10]. During UGT, trajectories of the COP show a larger medial-lateral range, which may lead to a decrease in medial-lateral stability [28].

According to the correlations between muscle strength levels and body stability, it can be found that the foot rotation of the individual with stronger muscle 
power has a modification effect during UGT. Meanwhile, muscle strength and muscle power of male subjects showed significant negative correlation with the range of foot balance and ML-COP during UGT. However, for female subjects, the significant correlation mainly focused on their muscle power level. Our other finding is that compared with PGT, the correlation between muscle strength level and stability was more obvious during UGT. Data from the current study suggest that the lower limbs muscle strength levels including muscle strength and power might be correlated with body stability and foot balance during UGT and PGT. This result is consistent with the previous suggestion by Sarah et al. [18], [19] that patients should develop appropriate muscle strength and power to improve the ability to stop quickly and efficiently.

However, several limitations should be considered. Firstly, the subject's walking speed is not strictly regulated, which may demonstrate differences of termination mechanics [2], [19], and we originally intend to make the subject show the natural motion state as much as possible. Secondly, as our study did not carry out further subdivision and analysis of gait subphases, further studies for comprehensive data are required. And lastly, the sample size of this study was relatively small, which should be expanded in further study.

\section{Conclusions}

The overall findings from this study suggest that two types of gait termination have significant differences in the plantar pressure distribution, and in comparison, UGT poses greater challenges to the stability of human body, especially for women. At the same time, data from the current study suggests that that there might be a correlation between individual muscle strength levels and the stability during the gait termination, especially between the muscle power and UGT, which means that the more excellent muscle power an individual has, the more stable the body will be when UGT is performed. The results of this study may provide insights into strengthening the link between basic research and practical applications, such as specific sports training, rehabilitation assessment and treatment, clinical diagnosis, efficacy evaluation.

\section{Acknowledgements}

This study was sponsored by National Natural Science Foundation of China (No. 81772423), National Key R\&D Program of
China (2018YFF0300903) and K. C. Wong Magna Fund in Ningbo University.

\section{References}

[1] Bishop M., Brunt D., KukulKa C., Tillman M.D., PATHARE N., Braking impulse and muscle activation during unplanned gait termination in human subjects with parkinsonism, Neuroscience Letters, 2003, 348 (2), 89-92, DOI: 10.1016/S0304-3940(03)00738-9.

[2] Bishop M., Brunt D., Pathare N., Patel B., The effect of velocity on the strategies used during gait termination, Gait and Posture, 2004, 20 (2), 134-139, DOI: 10.1016/ j.gaitpost.2003.07.004.

[3] Bishop M., Brunt D., Pathare N., Patela B., The interaction between leading and trailing limbs during stopping in humans, Neuroscience Letters, 2002, 323 (1), 1-4, DOI: 10.1016/ S0304-3940(01)02525-3.

[4] Bonnet C., Carello C., Turvey M., Diabetes and Postural Stability: Review and Hypotheses, Journal of Motor Behavior, 2009, 41 (2), 172-192, DOI: 10.3200/JMBR.41.2.172-192.

[5] Cen X., Liang Z., GaO Z., Lian W., Wang Z., The Influence of the Improvement of Calf Strength on Barefoot Loading, Journal of Biomimetics, Biomaterials and Biomedical Engineering, 2019, 40, 16-25, DOI: 10.4028/www.scientific.net/ JBBBE.40.16.

[6] Chatzistergos P.E., Healy A., Naemi R., Sundar L., RAMaChandran A., ChOCKAlingam N., The relationship between hallux grip force and balance in people with diabetes, Gait and Posture, 2019, 70, 109-115, DOI: 10.1016/ j.gaitpost.2019.02.020.

[7] Hass C.J., Waddell D.E., Fleming R.P., Juncos J.L., GREGOR R.J., Gait Initiation and Dynamic Balance Control in Parkinson's Disease, Archives of Physical Medicine and Rehabilitation, 2005, 86 (11), 2172-2176, DOI: 10.1016/ j.apmr.2005.05.013.

[8] Ilmane N., Croteau S., Duclos C., Quantifying dynamic and postural balance difficulty during gait perturbations using stabilizing/destabilizing forces, Journal of Biomechanics, 2015, 48 (3), 441-448, DOI: 10.1016/j.jbiomech.2014.12.027.

[9] JaEger R.J., VAnitchatchavan P., Ground reaction forces during termination of human gait, Journal of Biomechanics, 1992, 25 (10), 1233-1236, DOI: 10.1016/0021-9290(92)90080-K.

[10] KIRBY K.A., Subtalar Joint Axis Location and Rotational Equilibrium Theory of Foot Function, Journal of the American Podiatric Medical Association, 2001, 91 (9), 465-487, DOI: $10.7547 / 87507315-91-9-465$.

[11] Kobayashi Y., Hobara H., Heldoorn T.A., Kouchi M., Mochimaru M., Age-independent and age-dependent sex differences in gait pattern determined by principal component analysis, Gait and Posture, 2016, 46, 11-17, DOI: 10.1016/ j.gaitpost.2016.01.021.

[12] LEE Y.J., Changes in the symmetry of external perturbations affect patterns of muscle activity during gait initiation, Gait and Posture, 2019, 67, 57-64, DOI: 10.1016/j.gaitpost.2018.09.023.

[13] MAKi B.E., MCILROY W.E., Control of rapid limb movements for balance recovery: age-related changes and implications for fall prevention, Age and Ageing, 2006, 35 (Suppl. 2), ii12-ii18, DOI: 10.1093/ageing/afl078.

[14] Ni M., Signorile J.F., Balachandran A., PotiaumPai M., Power training induced change in bradykinesia and mus- 
cle power in Parkinson's disease, Parkinsonism and Related Disorders, 2016, 23, 37-44, DOI: 10.1016/ j.parkreldis.2015.11.028.

[15] NuRse M.A., NigG B.M., The effect of changes in foot sensation on plantar pressure and muscle activity, Clinical Biomechanics, 2001, 16(9), 719-727, DOI: 10.1016/ S0268-0033(01)00090-0.

[16] Paul S.S., Canning C.G., Sherrington C., Fung V.S.C., Reduced muscle strength is the major determinant of reduced leg muscle power in Parkinson's disease, Parkinsonism and Related Disorders, 2012, 18 (8), 974-977, DOI: 10.1016/ j.parkreldis.2012.05.007.

[17] Polcyn A.F., Lipsitz L.A., Kerrigan D.C., Collins J.J., Age-related changes in the initiation of gait: Degradation of central mechanisms for momentum generation, Archives of Physical Medicine and Rehabilitation, 1998, 79 (12), 1582-1589, DOI: 10.1016/S0003-9993(98)90425-7.

[18] Ridge S.T., Henley J., Manal K., Miller F., Richards J.G., Kinematic and kinetic analysis of planned and unplanned gait termination in children, Gait and Posture, 2013, 37 (2), 178-182, DOI: 10.1016/j.gaitpost.2012.06.030.

[19] Ridge S.T., Henley J., Manal K., Miller F., Richardset J.G., Biomechanical analysis of gait termination in 11-17 year old youth at preferred and fast walking speeds, Human Movement Science, 2016, 49, 178-185, DOI: 10.1016/ j.humov.2016.07.001.

[20] Roeing K.L., Moon Y., Sosnoff J.J., Unplanned gait termination in individuals with multiple sclerosis, Gait and Posture, 2017, 53, 168-172, DOI: 10.1016/j.gaitpost.2017.01.016.

[21] Sparrow W.A., Tirosh O., Gait termination: a review of experimental methods and the effects of ageing and gait pathologies, Gait and Posture, 2005, 22(4), 362-371, DOI: 10.1016/j.gaitpost.2004.11.005.
[22] Sun D., Gu Y., MeI Q., Shao Y., Sun J., Fernandez J., Effect of Heel Heights on Female Postural Control During Standing on a Dynamic Support Surface With Sinusoidal Oscillations, Journal of Motor Behavior, 2017, 49 (3), 281-287, DOI: 10.1080/00222895.2016.1191423.

[23] Tirosh O., Sparrow W.A., Age and walking speed effects on muscle recruitment in gait termination, Gait and Posture, 2005, 21 (3), 279-288, DOI: 10.1016/j.gaitpost.2004.03.002.

[24] Wearing S.C., URry S., Smeathers J.E., Battistutta D., A comparison of gait initiation and termination methods for obtaining plantar foot pressures, Gait \& Posture, 1999, 10 (3), 255-263, DOI: 10.1016/S0966-6362(99)00039-9.

[25] Wojciechowski E., Sman A., Cornett K., Raymond J., Refshauge J., Menezes M.P., BuRns J., Gait patterns of children and adolescents with Charcot-Marie-Tooth disease, Gait and Posture, 2017, 56, 89-94, DOI: 10.1016/ j.gaitpost.2017.05.005.

[26] Yan S.H., Zhang K., TAN G.Q., JiN Y., LiU Z.C., Effects of obesity on dynamic plantar pressure distribution in Chinese prepubescent children during walking, Gait and Posture, 2013, 37 (1), 37-42, DOI: 10.1016/j.gaitpost.2012.05.018.

[27] Zhang X., Li B., Hu K., Wan Q., Ding Y., Vanwanseele B., Adding an arch support to a heel lift improves stability and comfort during gait, Gait and Posture, 2017, 58, 94-97, DOI: 10.1016/j.gaitpost.2017.07.110.

[28] Zhang X., Li B., Influence of in-shoe heel lifts on plantar pressure and center of pressure in the medial-lateral direction during walking, Gait and Posture, 2014, 39 (4), 1012-1016, DOI: 10.1016/j.gaitpost.2013.12.025.

[29] ZhANG Y., Lu H., Gu Y., Hu N., Characteristics of the centre of pressure progression for pregnant women during walking, International Journal of Biomedical Engineering and Technology, 2015, 17(4), 387-397, DOI: 10.1504/IJBET.2015.069406. 